\title{
Remarques sur la surprise, la méprise et la déprise
}

\author{
Jean-Luc Marion
}

\section{OpenEdition}

Journals

Édition électronique

URL : http://journals.openedition.org/alter/410

DOI : 10.4000/alter.410

ISSN : 2558-7927

\section{Éditeur :}

Association ALTER, Archives Husserl (CNRS-UMR 8547)

\section{Édition imprimée}

Date de publication : 1 décembre 2016

Pagination : $15-30$

ISBN : 978-2-9550449-2-6

ISSN : 1249-8947

Référence électronique

Jean-Luc Marion, «Remarques sur la surprise, la méprise et la déprise », Alter [En ligne], 24 | 2016, mis en ligne le 01 décembre 2017, consulté le 04 mai 2019. URL : http://journals.openedition.org/alter/410 ; DOI : 10.4000/alter.410 


\section{REMARQUES SUR LA SURPRISE LA MÉPRISE ET LA DÉPRISE ${ }^{1}$}

Jean-Luc Marion

Il m'est agréable de pouvoir m'appuyer sur ce que Claudia Serban vient de dire, non seulement parce qu'elle l'a peut-être mieux exposé que je ne l'avais moi-même fait, mais parce qu'elle me dispense, en partie du moins, de me répéter. Gratitude de la paresse, plus encore que de la vanité.

Revenons pourtant un instant sur cette salle où se tient notre séminaire. Apparemment, réellement donc, il s'agit d'une salle de cours, une salle de conférence, comme tant d'autres à l'École et dans tous les bâtiments de la rue d'Ulm. Il s'agit d'un objet, qui peut (et doit) se décrire comme tel : subsistant dans une présence permanente (selon la Vorhandenheit), avec les mêmes dimensions d'étendue et de forme, du moins pour un temps (jusqu'à la prochaine réfection de l'immeuble, et depuis la dernière). Cet objet peut (et doit) aussi être considéré comme un objet d'usage (selon la Zuhandenheit) : c'est un endroit destiné à ce qu'on puisse faire des cours, y travailler en séminaires, voire y discuter sans fin en assemblées très générales, aussi longtemps du moins qu'il y aura des études universitaires (ce qui n'a pas les promesses de l'éternité). Et pourtant cette salle peut (et doit) aussi se considérer non pas comme un objet, mais comme un événement, en plus d'une acception. Car, quand nous arrivons à et entrons dans cette salle, il s'agit d'un lieu re-baptisé du nom de « salle Celan », de ce poète roumain de langue allemande exilé en France,

\footnotetext{
${ }^{1}$ Cet article reprend et complète le texte d'une communication faite le 19 décembre 2013 dans le séminaire «Émotions et volitions : la surprise » organisé par Natalie Depraz à l'École normale supérieure, où notre propos fut précédé par une présentation introductive faite par Claudia Serban et reprise dans ce dossier sous le titre « Surprise et saturation ».
} 
aujourd'hui célèbre, mais qui enseignait, inconnu encore, dans les années 1960-70 à l'École, dans cette salle ou une autre (je l'ai oublié) ; il y donnait, avec un succès pédagogique d'ailleurs assez faible, des cours d'allemand élémentaire pour les "grands débutants », dont j'étais alors. Le fait de me retrouver aujourd'hui, avec vous, dans la salle Celan constitue donc, pour moi du moins, en quelque manière un événement: retour dans le passé, qui redevient non seulement présent plus encore qu'il ne fut sur le moment (car je ne voyais rien de bien remarquable dans cette salle et ce cours) ; plus encore, retour d'un passé qui ne prend son sens et sa portée que rétrospectivement, repris dans la surprise qui me déprend et où je comprends ce qui $\mathrm{m}^{\prime}$ arrivait un peu mieux aujourd'hui qu'aux jours lointains où cela m'arrivait pourtant au présent. Cette salle, en tant qu'elle provient du nom «Celan », me surprend donc comme un événement.

Mais cela ne vaut pas que pour moi, suivant l'anecdote de mon histoire individuelle. Car toute salle peut apparaître aussi et même $\mathrm{d}$ 'abord comme un événement. Car personne n'entre dans une salle tout uniment, par un simple déplacement dans l'étendue, puisque même pour se déplacer d'un point à un autre dans l'étendue, je dois avoir une raison et un motif pour le faire ; j'entre dans la salle, parce que quelque chose va y arriver et donc $m^{\prime}$ y arriver. La salle contient d'ailleurs déjà en elle-même des motifs d'y venir, selon sa destination (salle de cours, salle à manger, salle du conseil, salles des assises, etc.). Et je ne m'attends pas à ce qu'il m'arrive la même sorte de chose lorsque que j'entre dans un palais romain ou dans une prison, dans une église ou un stade. Mais, en sus de ces destinations déjà inscrites dans les pierres et les murs, chaque salle recèle un enjeu potentiel : le cours (bon ou mauvais), le repas (infect ou délicieux, cordial ou glacial), le vote d'un budget ou d'une nomination (favorable ou non), le verdict d'un procès (acquittement ou condamnation). En passant la porte de la salle Celan, nous savions chacun à quoi nous attendre (sur les interventions du séminaire, nous avions tous notre petite idée, sur la question à l'affiche aussi), mais en fait nous n'avons franchi cette porte que parce que nous ne savions pas à quoi nous attendre, du moins pas totalement; on pourrait même dire que nous ne nous sommes déplacés, d'un effort gratuit, plutôt que de rester chez nous pour y travailler (ou non), que parce que nous espérions ne pas savoir à quoi nous attendre. Nous sommes venus dans l'espoir d'une surprise. Ce qui implique que la salle Celan nous apparaît non pas comme un objet, mais potentiellement, en puissance comme un événement; mais précisément un événement se définit par sa possibilité, au-delà de l'effectivité. En ce sens, rien de plus banal - et c'est un point sur 
lequel on ne saurait trop insister - que l'événement, confirmant luimême la banalité de la saturation : la saturation des significations par l'excès de l'intuition ne concerne pas seulement une région étroite de phénomènes exceptionnels (comme le sublime chez Kant ou l'idée d'infini selon Descartes, pour exemple), mais bien tout ce qui peut nous mettre face à un événement - tel un pavé qui dépasse dans la cour d'un hôtel particulier du faubourg Saint-Germain. Au point que n'importe quoi peut fonctionner comme un événement, pourvu que son avènement nous surprenne - nous prenne par surprise.

Nous pouvons désormais revenir à la question de la surprise, parce que nous avons déterminé au moins le type de phénoménalité à laquelle elle correspond, pour ne pas dire à laquelle elle répond : la surprise se définit comme la tonalité affective que je prends et qui me prend, quand un phénomène surprend sur le mode d'un événement. Il faut bien voir que, dans le cadre des études sur la phénoménologie des impressions, des émotions, voire des sensations, la surprise offre un cas particulier - cas très particulier même, puisqu'il a partie liée, et d'une certaine manière depuis le début grec de la philosophie, à un débat initialement ouvert sous un autre nom que le sien : la surprise, sous le nom du thaumazein, autant dire de la stupéfaction (au-delà même de l'étonnement), devant l'interrogation aporétique qu'adresse la chose elle-même à laquelle on ne s'attendait pas. La surprise ou ce à quoi, jamais, nul ne s'attend. Ce lien entre surprise et thaumazein, Descartes l'affirme très clairement, en deux textes au moins : "L'admiration est une subite surprise de l'âme, qui fait qu'elle se porte à considérer avec attention les objets qui lui semblent rares et extraordinaires » (Passions de l'âme, § 70). Et aussi : «...elle [scilicet l'admiration] a beaucoup de force à cause de la surprise, c'est-à-dire de l'arrivement subit et inopiné de l'impression qui change le mouvement des esprits; laquelle surprise est propre et particulière à cette passion » (Passions de l'âme, § 72). Ces deux formulations nous apprennent déjà beaucoup. Premièrement, il apparaît que le secret de l'admiration se trouve dans la surprise, dont elle provient, et non pas l'inverse ; bref, l'admiration doit se concevoir à partir de la surprise, et tout refus de l'admiration implique, dans son fond, un refus de la surprise - une décision de ne jamais se laisser surprendre, de toujours contrôler la prise de la pensée, de toujours penser afin de comprendre et en contrôlant, de ne jamais laisser le phénomène prendre la mesure de ma prise de vue pour $\mathrm{m}^{\prime} \mathrm{y}$ imposer une déprise de ma pensée à sa démesure. Deuxièmement, la surprise, comme son nom l'indique très bien en français, "prend » celui qui l'éprouve ; en fait, ici le verbe «prendre » change de sens et vire sur lui-même : " prendre » ne veut 
plus dire "comprendre », mais indique la situation de «ne pas comprendre ». Ou plus encore, la surprise recommande inversement non seulement de ne plus exercer de compréhension, mais de la subir, de $\mathrm{s}^{\prime} \mathrm{y}$ laisser reprendre et déprendre. La surprise est le fait de penser sans exercer une compréhension, mais en se déprenant de la préhension de l'événement qui arrive à la pensée.

Une telle inversion originaire mérite d'être notée: la surprise implique et provoque une attitude de pensée essentiellement passive. Nul hasard à ce qu'elle suscite rien de moins que la première passion aux yeux de Descartes, l'admiration, mais qu'elle aboutisse aussi à la fin, du fait du lien entre admiration et générosité, à la plus haute vertu, cette générosité même. Or cette soumission se trouve marquée par le fait que la "surprise», qui surprend inopinément l'âme, advient comme un événement, ou plus précisément survient par un «arrivement» subi (Passions de l'âme, §72); et ce terme d' «arrivement» reste, à notre connaissance, un hapax chez Descartes, et d'usage très rare en français moderne (à peine mentionné en français moyen, ignoré des dictionnaires contemporains). Faute de cet usage, j'ai ailleurs préféré remettre en service le terme d' «arrivage», en le détournant de son emploi habituel, restreint à la livraison de la marée chez les poissonniers : l'arrivage n'indique en effet pas seulement la livraison (delivery, dit l'anglais) de la pêche sur les étals de la ville et sur les tables des restaurants, mais surtout que cette livraison ne peut se prévoir, ni dans son contenu, ni dans son heure ou sa date, parce qu'elle dépend du flot et du jusant, de l'état de la mer et du temps, du succès de la pêche, des aléas du transport, etc. L'arrivage (donc aussi $l^{\prime}$ «arrivement») ne peut se prévoir, ni se comprendre, ni, en toute rigueur, s'attendre. Ou plutôt, je dois m'attendre à ce qu'arrive ce que je n'attendais précisément pas, c'est-à-dire un événement au sens le plus radical, non prévisible, qui arrive par surprise, quand il le veut bien, quand « cela lui chante », comme la mer qui surprend l'homme, et que l'homme jamais ne comprend, surtout pas par la pensée. $\mathrm{L}^{\prime}$ « arrivement» de l'arrivage pourrait se rapprocher de ce que Heidegger entend parfois par l'Überkommnis, telle qu'il la distingue de l'Ankunft. Dans ce dernier cas, il s'agit de l'arrivée prévue et affichée d'un horaire, par exemple de l'arrivée d'un train en gare ou d'un avion à l'aéroport, avec une ponctualité dont la compagnie ferroviaire ou aérienne peut, si elle y parvient, tirer un argument de vente. Au contraire, dans l'autre cas, l'arrivage n'arrive jamais au moment précis d'un horaire, parce que ce moment, seul l'arrivage luimême le détermine. Et la vigueur, la rigueur et la puissance de l'événement tient justement à ce que lui seul peut fixer le jour et 
l'heure de son accomplissement, jour et heure que tout autre ignore et doit ignorer, faute de quoi il ne s'agirait plus d'un événement. Que la souveraine autorité de l'événement ne paraisse pas avec évidence, ou que cette évidence ne dure pas, Heidegger l'a parfaitement exposé : dans l'événement, où se déploie la vérité, l'arrivée ponctuelle (Ankunft) de l'étant ne peut pas ne pas occuper toute la visibilité sur la scène phénoménale; quant à l'arrivage ( $l^{\prime}$ « arrivement » ou l'Überkommnis), où s'opère le déploiement de l'être, lui, ne se voit pas, offusqué qu'il ne peut pas ne pas devenir par l'étant, dont l'arrivée ponctuelle et factuelle efface, pour ainsi le dire, l'advenir et l'avènement qui l'a rendu possible. L'arrivage se passe et donc il passe, dépassé par le fait (lui, arrêté) de l'arrivée. Ainsi, l'arrivage qui surprend ne le peut que parce qu'il (se) passe et donc disparaît aussi vite $\mathrm{qu}^{\prime} \mathrm{il}$ se produit. La surprise ne peut donc durer plus que lui.

L'admiration s'avère une affaire de surprise. Et comme la surprise, elle ne persiste pas dans la présence endurante. Elle ne se passe qu'autant qu'elle passe et consiste en ce passage. Mais cette première caractéristique, qui semble indiscutablement négative du point de vue de la métaphysique (où, précisément, être consiste à persister, donc à ne pas passer), conduit à définir, en un second moment, le privilège de la surprise, ou plutôt de ce qui la rend possible. La surprise advient par surprise, contre toute attente, puisqu'en effet l'arrivage de l'événement ne peut ni ne doit se prévoir. Elle se déploie donc non seulement sans prévision, mais à la condition de son imprévisibilité, contre toute attente. Il lui appartient par principe de s'accomplir de telle sorte qu'elle réduise à quia la demande pour un quod, un warum ou un parce que qui rendraient raison et cause de son advenue, comme de sa disparition. La surprise, donc aussi l'arrivage qui la provoque, se soustraient l'une et l'autre à l'activité de la moindre cause, donc à la compréhension d'un sujet éventuellement constituant ou objectivant. La surprise suppose, mais comme un privilège, de se produire seulement sur fond d'ignorance - entendue au sens précis de l'ignorance de tout objet que l'on pourrait prévoir sur le fond de concepts a priori. Ce qui ne signifie pas que la surprise et son arrivage ne se peuvent pas penser, ou qu'ils ne se conçoivent pas; mais seulement qu'ils ne se conçoivent pas comme un objet compréhensible, parce que prévisible ; ou plus exactement, qu'ils ne se conçoivent qu'à condition de ne pas se laisser comprendre comme un objet. Il convient au contraire de les penser à partir de la caractéristique fondamentale de tous les phénomènes saturés, l'événementialité. Ici le défaut et la force de la surprise coïncident : c'est parce que nous ne savons pas, que la surprise nous affecte. Et sur ce 
point aussi, Descartes prend une position claire, sans ambiguïé, évoquant des "...choses que nous avons auparavant ignorées. Car nous n'admirons que ce qui nous paraît rare et extraordinaire, et rien ne peut nous paraître tel, que pour ce que nous l'avons ignoré, ou même aussi pour ce qu'il est différent des choses que nous avons sues» (Passions de l'âme, §75). Ou bien: "Lorsque la première rencontre de quelque objet ${ }^{2}$ nous surprend, et que nous le jugeons être nouveau ou fort différent de ce que nous connaissions auparavant, ou bien de ce que nous supposions qu'il devait être, cela fait que nous l'admirons et en sommes étonnés » (Passions de l'âme, § 53). Il peut s'agir d'une ignorance définitive, ou, plus fréquemment, d'une ignorance provisoire, qui s'éteindra lorsque l'étonnement le cédera à la reconnaissance d'objets là où, d'abord, n'apparaissaient que des événements. En ce sens, le progrès des sciences se mesure au décès de la surprise. Mais il se paie à un prix épistémologique élevé : il faut admettre ne connaître plus que ce que l'on peut comprendre, des objets précisément, qui n'enrichissent notre science qu'à la condition expresse de ne jamais y admettre de l'inconnu. En termes de métaphysique, il n'y a pas lieu d'admettre ce qui se soustrait à l'anticipation. Mais la surprise interdit l'anticipation, parce qu'elle réclame l'arrivage comme norme. Au risque de simplifier (mais, à la fin, il faut toujours en finir par simplifier), la surprise rend possible la philosophie, mais devient impossible en métaphysique.

Mais il y a plus: Descartes détermine ici déjà ce que Kant nommera l'anticipation, opposant la surprise à «...ce que nous connaissions auparavant, ou bien de ce que nous supposions qu'il devait être ». Dans la surprise, ce qui nous advient par «arrivement» contredit ce que nous attendions, la chose n'apparaît pas comme elle aurait d $\hat{u}$. Kant, décrivant précisément les anticipations de la perception, ou plus généralement les « anticipations des phénomènes (der Erscheinungen) » (Critique de la raison pure, A 167), distingue entre le caractère formel de l'anticipation (les formes pures de l'espace et du temps, donc, au regard de la forme, des grandeurs) et son caractère matériel, «la perception (comme matière de la sensation) » qui, elle, ne peut s'anticiper. Ainsi, l'anticipation ne concerne, dans le phénomène, que le degré de la sensation, grandeur intensive de la perception. Et Kant de conclure, évidemment, qu'il n'y a une anticipation de la perception que formellement, tandis qu'on ne peut anticiper (sur) la sensation. Dans ce contexte, comment définir la

\footnotetext{
${ }^{2}$ Le terme étant à entendre ici au sens le plus large et vague, précisément pas celui que nous retenons lorsque nous l'opposons à l'événement.
} 
surprise? Bien entendu, comme ce qui ne peut s'anticiper - mais doublement. D'abord parce que la surprise intervient quand arrive la " matière de la sensation » (surprise pour ainsi dire matérielle) ; mais aussi parce que, dans la surprise, au contraire de l'anticipation, la « matière de la sensation » contredit ce que l'anticipation attendait, ou plutôt ce à quoi elle s'attendait (surprise pour ainsi dire formelle). Dans la surprise, la perception échappe ainsi deux fois à l'anticipation. On comprend mieux, au vu de cette double destruction de l'anticipation par la surprise, que l'admiration, qu'elle rend possible, puisse apparaître comme une peur et l'inspirer, conformément à la définition, elle-même surprenante mais profondément juste, qu'en propose saint Thomas d'Aquin : " ...admiratio est species timoris, consequens apprehensionem alicujus rei excedentis nostram facultatem » (Summa theologiae, IaIIae, q. 180, a. 3, ad 3m). Ce qu'on peut évidemment traduire par: "L'admiration c'est une forme de la peur, et de la peur devant l'excès de ce qui est à connaître »; mais où l'on doit sans doute et finalement entendre qu'elle est une sorte de peur, qui résulte de notre appréhension de ne pouvoir appréhender par anticipation une chose, parce qu'elle excède, comme un phénomène saturé, notre faculté d'appréhender. On ne saurait, en effet, mieux dire : l'admiration, qui prend la connaissance par surprise, fait peur.

Peur devant une conséquence directe et paradoxale : l'admiration intervient comme première figure de la conscience (et, en ce sens, comme la première des passions) en tant qu'elle reste passive, puisqu'elle détermine le « je », l'egocogitans en tant qu'il s'éprouve ne connaissant pas, pensant certes, mais précisément en tant que ne pouvant pas connaître. Ce qui, d'ailleurs, rapproche l'admiration du doute - ils partagent l'inconnaissance dans la pensée, ou même le premier mode de la cogitatio. Ce qui manifeste, à tout le moins, qu'un « je » peut penser sans pour autant exercer la fonction d'anticipation. À l'encontre de Kant, certes, mais aussi de Descartes, dont les «natures très simples (naturae simplicissimae)» des Règles pour la direction de l'esprit prétendaient déjà prévoir toujours les déterminations de l'expérience, quels qu'en soient le contenu et la matière. Au contraire, dans le cas de la surprise et de l'admiration qu'elle rend possible, l'impossibilité de connaître n'entraîne pas que rien ne se passe, mais seulement que rien de connaissable ne se passe. Ou plutôt, même si rien de reconnaissable par anticipation et de connaissable comme un objet ne se passe, cependant, je pense et donc suis. Je pense, même si je ne pense pas selon les exigences de l'attitude théorique.

Cette peur explique aussi pourquoi le débat sur l'admiration fut si central et ardent, durant la période qui va au moins de saint Thomas 
à Kant (notons que cela ferait un utile sujet de thèse). L'enjeu en fut très clairement exprimé par sa critique chez Malebranche, chez Leibniz, et d'abord et surtout de la manière la plus exemplaire chez Spinoza. Quand ce dernier critique l'admiration, c'est pour une raison très précise et qui anticipe sur Kant: alors que dans les autres imaginations, l'esprit ne reste fixé (defixa, detineri) sur une pensée (la pensée d'un mode ou d'un ensemble de modes) qu'aussi longtemps que n'interviennent les images (imaginations) d'autres choses liées (concatenae \& ita ordinatae) avec la première, selon une relation strictement causale ( $a b$ aliis causis), dans le cas de l'admiration, au contraire, l'esprit reste fixé sur une chose, sans que d'autres imaginations ne parviennent, à titre de causes, à l'en distraire. L'esprit se distrait des autres choses et images, parce que cette idée, qu'il admire, le retient, sans que pourtant aucune cause n'intervienne ("...haec Mentis distractio ex nulla causa positiva [...] oritur », Éthique III, définition IV). Ou plus exactement, l'admiration retient l'attention de l'esprit en le soustrayant de l'enchaînement de ses pensées (imaginations) par une connexio causale (l'ordo $\mathcal{E}$ connexio aussi bien rerum qu'idearum) ; donc, avec l'admiration, non seulement l'esprit pense sans cause, mais il pense en se libérant, lui et sa pensée, de la causalité. D’où Spinoza conclut qu'elle ne constitue pas le premier des affectus (la première des passions), mais qu'il faut en quelque manière l'exclure de la liste, conclusion excessive d'une différence bien vue: en effet, cette distraction de l'esprit née d'une absence de cause positive, d'un «...deficit » (Éthique III, définition IV), fait de l'admiration une idea ni adéquate, ni même inadéquate, parce qu'elle échappe à ce qui autorise cette dichotomie, à savoir, le lien causal entre chaque idée et la précédente ou la suivante, lien causal correct dans un cas, incorrect dans le cas de l'imagination, où les effets ignorent leurs causes. Spinoza anticipe ainsi sur Kant exposant les catégories de la relation : la connaissance d'un phénomène implique toujours de lier celui-ci à un autre par rétablissement d'une relation, en l'occurrence causale ; et, si possible, une relation adéquate (autant de réalité dans la cause que dans l'effet, par exemple). Dans l'imagination, nous ne parvenons pas à cette relation adéquate; et le pire cas d'imagination se trouve dans l'admiration où nous restons fichés (pas seulement fixés) sur une seule chose, un phénomène hors chaîne causale, surgi de nulle part, qui ne veut pas disparaître, bref, un événement. Or, en stricte metaphysica, on ne saurait absolument pas connaître un phénomène isolé ; le connaître implique toujours de le relier à un autre phénomène, par un rapport soit d'accident à substance, soit de substance à substance, ou bien par un rapport de cause et d'effet. Ainsi, l'admi- 
ration, qui surgit de la surprise, mettrait en cause, ou plutôt en suspens, le principe de causalité, c'est-à-dire, en dernière instance, le principium reddendae rationis.

Il faut donc admettre que l'affaire est sérieuse, au point que la surprise (et les excès qu'elle provoque dans l'étonnement, qui fait durer l'admiration, cf. Passions de l'âme, §76) mettrait en question l'accomplissement même du système de la metaphysica. Mais prendre congé de la metaphysica n'équivaut pas à quitter ni à se détourner de la philosophie. Au contraire, en fait, puisque, tant pour Platon que pour Aristote, la philosophie commence avec le thaumazein, c'est-àdire avec la stupéfaction, la surprise, l'étonnement, l'admiration

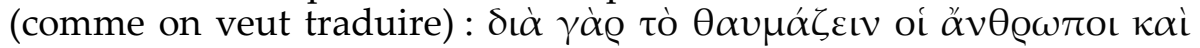

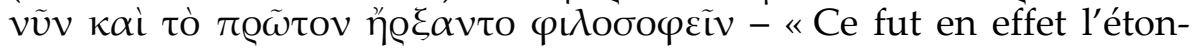
nement qui poussa, aujourd'hui comme la première fois, les hommes à philosopher » (Métaphysique, A, 2, $982 \mathrm{~b}$; la formulation est assez solennelle, pour penser à $Z, 1,1028 b)$. Il s'agit en tout cas d'un écho à

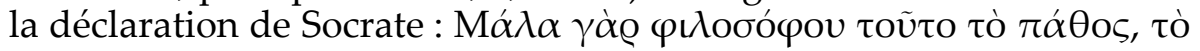
$\theta \alpha v \mu \alpha ́ \zeta \varepsilon \iota v$ - «Voilà bien là l'affect d'un philosophe, admirer! » (Théétète 155d). Pourquoi donc? Parce que la philosophie commence non point par la connaissance théorique du monde, mais par le souci de la "merveille des merveilles », que le monde soit, ou plutôt apparaisse. Il se pourrait donc que la question de la surprise, jusqu'à la Bewunderung de Kant devant la raison pratique, offre le symptôme, le plus souvent méconnu, d'une réforme de l'entendement secrètement possible, quoi que jamais explicitement envisagée - non une réforme de l'entendement, mais, tout à l'inverse, une réformation de l'ego hors de la primauté de l'entendement en lui et dans sa pensée. La surprise pense sans et avant l'entendement, comme aussi l'estime et le doute. Nous atteignons ici un endroit (un moment aussi) où se décide quelque enjeu crucial. Car quand l'ego se laisse déterminer par la surprise, il n'aborde plus le monde, ni lui-même dans un rapport théorétique, ni comme une totalité d'objets à constituer. Et depuis, de Nietzsche à Wittgenstein, de Husserl à Heidegger, la philosophie n'a cessé, en un sens encore à exposer, de méditer cette fonction ou cette exigence de la surprise.

De cette première remarque, il s'ensuit une deuxième question : avec la surprise nous n'aurions donc pas affaire d'abord à une théorie des passions, ou du sentiment en général, ni même à une théorie morale. Il s'agirait de la suspension de l'attitude théorétique face au monde, ce qui implique donc aussi la suspension du clivage, supposé bien connu, mais en fait hautement problématique, entre l'attitude théorétique et l'attitude pratique. La surprise aurait affaire avec le 
doute - à une réserve près : car si le doute a pu apparaître comme une quasi-réduction, il reste à l'intérieur de l'attitude théorétique. On peut $\mathrm{s}^{\prime}$ interroger pour savoir si la réduction husserlienne, d'une certaine manière, reste à l'intérieur de l'attitude théorétique et du primat de la théoria, alors que tel n'est évidemment pas le cas avec l'angoisse selon Heidegger ou avec le commandement d'autrui pour Levinas, qui ont clairement ambitionné d'instituer des quasi-réductions transgressant l'horizon théorétique. Mais la surprise a cette particularité que, d'une certaine manière, elle ne peut s'entendre que comme une suspension de l'attitude théorétique. En ce sens, elle offre un cas beaucoup plus impressionnant que, par exemple, ce contrepoint de l'établissement de la philosophie comme métaphysique que fut l'histoire parallèle du scepticisme ; car c'est la faiblesse du scepticisme (et ce pourquoi il n'a finalement jamais fait douter de rien) qu'il reste toujours conforme à l'idéal théorétique. Le scepticisme prétend démontrer que nous ne connaissons rien, et le démontrer certainement, voire par argumentation logique. Il veut donc encore connaître - d'où son manque de radicalité.

La surprise ne tente pas de se soustraire à l'attitude théorétique par une démarche critique ou sceptique, en ce sens encore théorétique, ne fût-ce qu'au prix d'une inversion. La surprise constate et éprouve au contraire que c'est la chose même qui apparaît sans se soumettre aux exigences objectives (ou objectivantes) de l'attitude théorétique. Revenons à Descartes pour concevoir mieux le «deficit» que stigmatisait Spinoza et étonnons-nous du vocabulaire qu'il utilise pour évoquer la surprise : «...tout le corps demeure immobile comme une statue et on ne peut apercevoir de l'objet que la première face qui s'est présentée, ni par conséquent en acquérir non plus une particulière connaissance» (Passions de l'âme, §75). Ou encore: "...lorsqu'elle est excessive et qu'elle fait qu'on arrête seulement son attention sur la première image des objets qui se sont présentés, sans en acquérir d'autre connaissance " (Passions de l'âme, § 78). Avouons notre surprise devant cette double description de la surprise (en fait de la surprise à son maximum et son excès, l'étonnement, la surprise au sommet de son étrangeté donc). D'abord, Descartes reconnaît que, dans ce cas, nous n'avons accès qu'à la "première face », la "première image » de la chose, telle qu'elle ne suffit pas à « ....acquérir une autre connaissance », " ...à en acquérir une plus particulière connaissance ». Ainsi ce que, surpris, nous apercevons de la chose reste si essentiellement partiel qu'aucune connaissance ne peut en résulter - aucune connaissance adéquate (ou du moins complète) d'un objet, aucune constitution d'un phénomène autour de son noyau noéma- 
tique. On croirait en effet entendre ici Husserl parler de l'appréhension d'un phénomène dans ses premières esquisses (Abschattungen); ce qui permet une première description phénoménologique, mais non pas la compréhension d'un objet. (Certes, Descartes parle bien ici d'un « objet », mais cette approximation ne vise pourtant que la première face de ce qui se présente, et dont précisément il ne dit pas que nous la connaissions.) Comment concevoir cette non-connaissance de simples «faces » et « images », en quoi leur imprécision reste-telle pourtant suffisamment forte pour imposer la stupeur d'une "statue»? La réponse ne semble pas douteuse aux yeux de Descartes: la chose, même mal ou pas connue, $\mathrm{m}^{\prime}$ immobilise au songe froid de l'étonnement parce que, sans aucun statut théorétique, mais peut-être d'autant plus puissamment, la chose qui survient se présente («...s'est présentée...», «...se sont présentés »). Ces termes, fort rares de trouver sous la plume de Descartes, ne signifient pas peu : la chose devient présente certes, mais non parce que, comme dans une situation théorétique normale (et méthodique), la mens lui imposerait ses conditions de présentification, a priori quant aux formes, d'une présence empruntée, dérivée et conditionnelle; mais parce que la chose impose à la mens (et la surprend donc) le surgissement de sa propre présence, qu'elle se donne et s'accorde selon ses propres exigences. Ainsi, la surprise, par son déficit même, délivre en quelque manière la chose du présupposé d'un horizon théorétique et lui permet de se présenter. La chose se trouve, en situation de surprise, en quelque manière, autorisée à se phénoménaliser - et ceci, même pour Descartes, qui est pourtant en un sens le dernier philosophe à pouvoir le faire, ou le premier à ne pas pouvoir le faire, à ne pas vouloir le faire. Donc pour qu'il puisse le faire, pour qu'il puisse décrire la surprise, il fallait qu'il y soit obligé. Concluons que la question de la surprise entretient sans doute un lien privilégié avec un tournant phénoménologique en philosophie.

Alors, il reste maintenant deux autres questions à aborder, l'une qui est le statut du « je » dans la surprise, et l'autre qui est l'hypothèse émise par Claudia Serban (que je découvre en même temps que vous), de savoir s'il y a un lien entre la surprise et la saturation. Et d'abord, que devient le «je » en situation de surprise? La surprise fait que le «je », avant même d'avoir rang d'un adonné, a le statut d'un « interloqué » (et c'est bien dans cet ordre que se sont découvertes les choses). Or on peut lier directement la surprise à $\mathrm{l}^{\prime}$ « interloqué ». Reste que l'adonné se définit aussi comme celui qui se découvre lui-même donné (et reçu) quand et au même moment où il reçoit un donné. L'adonné étant ainsi le premier empiriste, tandis que 
l'empiriste n'est jamais un, parce qu'il présuppose toujours le récepteur de l'empirisme comme lui-même non reçu (ni donné), déjà établi comme conscience d'apparence passive, mais d'autant plus dogmatique qu'elle s'excepte elle-même de la réception. Ou bien l'on peut procéder un peu autrement: partir de la surprise, donc de $l^{\prime}$ « interloqué », et se demander quelle est la figure du «je » qui permettrait de rendre visible l'ambiguïté de la surprise - cette impression dont on ne sait rien, mais qui s'impose précisément par ce déficit. Ici on peut convoquer l'appel et le témoin : l'appel d'abord, car son propre consiste en ce qu'on peut l'entendre non seulement sans en identifier le contenu, ni l'origine, ni même être absolument certain qu'il y a vraiment eu un appel (car là où il peut bien n'y avoir que l'impression d'un appel, il se produit souvent autant d'effet avec un appel rêvé qu'avec un appel confirmé, et un appel rêvé reste un appel pour celui qui le reçoit). Ainsi, l'appel partage-t-il une caractéristique essentielle de la surprise : en un sens, il n'a pas besoin d'un sens explicite et constitué pour s'exercer. Parce que même s'il n'a pas de sens confirmé par une validation extra-mentale, l'appel fonctionne encore comme un appel, exactement comme la surprise fonctionne d'autant mieux si celui qui se découvre surpris ignore par quoi il l'a été. Et si j'ai soutenu dès le début à l'anonymat de l'appel, ce n'était que pour m'en tenir au paradoxe (formulé, je crois, par Jean-Louis Chrétien pour la première fois) que l'appel ne s'entend que dans la réponse et que $c^{\prime}$ est la réponse qui dit qu'il y a eu un appel - et lequel. Toute la différence entre un appel et une simple proclamation ou une communication d'informations tient en ceci que l'appel ne trouve son sens plénier que dans la réponse, et ces autres, non. Tant qu'il n'y a pas eu de réponse, l'appel n'est toujours pas phénoménalisé. Ainsi, pour toutes ces raisons, l'appel reproduit des caractéristiques de la surprise.

Passons au dernier point: la surprise fait-elle signe vers l'événement ou vers la saturation? Il me semble que tout dépend du statut que l'on accorde à l'événement: l'événement se réduit-il à un objet historique, un objet historicisé, un objet transitoire ? Il semblerait que tel soit le plus souvent le cas. Pour le dire autrement, demandonsnous pourquoi la philosophie, qui s'est tant et si bien intéressée au temps et à l'histoire, a été si discrète sur la spécificité de l'événement ? Ceci même et jusque chez Heidegger, puisque que, dans Sein und Zeit, on aurait pu attendre une détermination de l'événement comme un étant qui n'est pas vorhanden, ni zuhanden, ni même peutêtre daseinsmässig. Et quant à lui, l'Ereignis ne revient à pas l'événement, du moins pas directement. Certes, entre toutes les différentes 
acceptions du terme, Heidegger a parfois aussi employé Ereignis dans son acception la plus banale ; mais justement, cette banalité, il ne l'a pas explicitée phénoménologiquement ; et surtout, il n'a jamais songé qu'il ne s'agissait peut-être pas d'une banalité ou d'une acception qu'il fallait supprimer, comme inutile, afin de refonder le sens neuf du terme. En d'autres mots, pourquoi Ereignis ne revient jamais chez Heidegger à l'événement? Par contraste, il me paraît que la vraie question à propos de l'événement tient à ce qu'il définit essentiellement tout phénomène saturé (et saturant) dans sa possibilité. Tenons en effet pour acquis que l'événement n'est pas un objet, parce qu'il ne respecte pas les catégories de la modalité. En effet, ces catégories, commençant par le possible, suivent la définition métaphysique du possible (qu'elle soit logique, ou qu'elle soit critique, cela ne change finalement pas tant sur le fond), à savoir, le possible est ce qui peut ou bien se concevoir logiquement sans contradiction (et telle est bien la définition de l'étant dans l'ontologie classique : ce qui se conçoit sans contradiction, cela seul peut être), ou bien - et c'est ici qu'intervient la modification apportée par Kant - est possible ce qui est conforme aux conditions formelles de l'expérience, donc : est possible ce qui peut advenir dans les conditions de l'expérience; mais cette radicalisation transcendantale ne fait, en un sens, que rendre explicite la vérité de la non-contradiction. Dans ces conditions, en principe tout objet, et donc tout événement (et c'est pourquoi il ne pouvait pas y avoir de pensée de l'événement dans sa spécificité), doit d'abord être possible avant que d'être effectif ; doit donc passer de la possibilité à l'effectivité, suivant un chemin normal et normé, qui offre le privilège de pouvoir être anticipé, parce que les conditions de possibilité sont connues a priori, donc par avance. Ainsi, non seulement l'effectif reste possible, pensable et non-contradictoire, mais, en retour, dès la possibilité, on sait qu'on suit la voie de l'effectivité ; il ne manque à la possibilité que la position pour poser le pied sur le sol de l'effectivité ; mais dans le développement technique de la métaphysique, la position devient le pas le plus facile à franchir. Ce qui constituait la difficulté (passer du possible à l'effectif) a disparu comme difficulté avec l'accomplissement technologique de la métaphysique, où il y a une chose que l'on sait toujours faire : passer du concept à la production. Et ceci, non pas pour des raisons ponctuelles et provisoires (la Chine remplaçant l'Angleterre comme manufacture mondiale), mais parce que la position se trouve elle-même en quelque manière déjà incluse dans la possibilité (sous le nom d'industrialisation, mais pas uniquement). La difficulté se trouve au contraire dans la définition du concept ; et, sitôt trouvé le concept, quasiment tout est fait : il ne reste 
qu'à industrialiser, puis à produire, puis à vendre. Donc l'effectivité se trouve de toute manière déjà contenue dans la possibilité, c'est-àdire dans la concevabilité.

$\mathrm{Au}$ contraire de l'objet et de ses trois catégories modales (possibilité, effectivité, nécessité), l'événement nous prend par surprise. Et il nous prend par surprise de plusieurs façons. Premièrement parce que l'événement atteint l'effectivité sans jamais passer par la possibilité : quand l'événement arrive, il demeure la plupart du temps et de prime abord inintelligible, et c'est pourquoi tout le monde en dit «mais c'est... c'est impossible»: car c'est bien de l'imprévisible qu'il s'agit. L'impossible devient effectif et va le rester bien qu'on ne le comprenne pas toujours, et qu'au mieux il puisse devenir rétrospectivement possible. Telle est la première façon dont l'événement résiste au primat de la possibilité, donc de l'attitude théorétique.

Qu'on n'objecte pas que cette impossibilité effective, l'événement va la perdre, puisque des savants experts viendront pour nous l'expliquer à force de théorie ou d'idéologie - car avec le temps, tout peut s'expliquer (ce qui est tout à fait exact, ou peut-être tout à fait exact). Admettons que les historiens déferont tout événement en un objet complexe ou un complexe d'objets: on va peut-être, rétrospectivement, trouver toutes les causes requises. Mais, précisément, ces causes et ces explications seront alors le résultat de l'effet, parce que, en réalité, dans le cas de l'événement, les causes sont toujours le résultat de l'effet, et pas l'inverse. C'est seulement pour les objets que nous avons des causes qui précèdent l'effet, mais jamais pour l'événement. $C^{\prime}$ est la deuxième façon de contredire le rapport entre le possible et l'impossible. Et il y a une troisième façon, qui est encore plus claire : c'est que, même inintelligible dans son effectivité, donc impossible, l'événement, et en particulier l'événement collectif (mais c'est aussi vrai pour un événement advenant à un seul individu), va définir de nouveaux possibles, c'est-à-dire que tout va s'adapter et se comprendre à partir de cet événement, pourtant resté inintelligible, impossible mais effectif. Ainsi par exemple, qu'est-ce qui s'est passé le 11 septembre 2001? Nous ne le savons pas, ni peut-être ne le saurons-nous jamais vraiment; mais, et cela nous le savons, si nous voulons comprendre ce qui se passe depuis le 11 septembre, il faudra analyser une grande partie des phénomènes à partir de ce point de repère. Ou encore, comment quelque chose de si étrange que la guerre de 1914-18 a pu arriver? Nous ne le savons pas encore, mais cette chose inconcevable devenue effective a pour cela même déterminé tous les possibles du siècle qui a suivi. Donc l'événement 
s'impose comme un impossible effectif, qui échappe au possible (entendu au sens de la prévision, de l'anticipation) et qui produit des possibles ou permet de les concevoir.

C'est alors que nous sommes vraiment dans le domaine de la surprise. Car la surprise n'est pas un moment, mais un rapport au monde, et un autre rapport au monde, où nous ne sommes pas ouverts au phénomène premièrement selon l'attitude théorétique (ce qui ne veut pas dire que ce soit une attitude purement pratique, puisque la distinction entre théorie et pratique ne vaut pas des événements). Et donc la question de la surprise rejoint le propre de l'histoire - il s'agit du temps, en sorte que ni l'une (la surprise), ni l'autre (l'histoire) ne peuvent se répéter ou se retourner. On ne peut pas revenir sur l'histoire, avec laquelle nous n'avons pas de relation d'abord théorétique ; Nietzsche, quand il faisait l'analyse du ressentiment, l'avait parfaitement compris. On ne revient pas sur l'histoire, et c'est pourquoi elle est affaire de surprises; nous sommes dans l'histoire selon la surprise. 